\title{
Baryon production at LHC energies and very high energy cosmic ray spectra
}

\author{
O.I. Piskounova ${ }^{\mathrm{a}}$ \\ P. N. Lebedev Physical Institute, Moscow, Russia
}

\begin{abstract}
The spectra of baryons at LHC can explain the features of CR proton spectra. It seems important to study all baryon data that are available from collider experiments in a wide range of energies. Transverse momentum spectra of baryons from RHIC $(\sqrt{(s)}=62$ and $200 \mathrm{GeV})$ and LHC experiments $(\sqrt{(s)}=0.9$ and $7 \mathrm{TeV})$ have been considered. It is seen that the slope of low $p_{T}$ distributions is changing with energy. The QGSM fit of distributions gives the average transverse momenta which behave approximately as $s^{0.06}$ that is similar to the previously observed behavior of $\Lambda$ baryon spectra. This slow growing of $<p_{T}>$ in hadron interactions of VHE in CR detectors cannot cause the "knee" in experimental proton spectra. In addition, the available data on $\Lambda_{c}$ production from LHCb at $\sqrt{s}=7 \mathrm{TeV}$ were also studied. The preliminary dependence of hadron average transverse momenta on their masses at the LHC energy is presented. The possible source of cosmic ray antiparticle-to-particle ratios that are growing with energy was also analyzed. The growing ratios are the result of local leading asymmetry for spectra of baryons and antibaryons that are produced in the kinematical region of proton target fragmentation. This asymmetry of baryon spectra, as they are converted into the energy distributions in the laboratory system, seems to result in an increasing ratio of secondary antiparticle-to-particle spectra up to a few hundreds of $\mathrm{GeV}$. This conclusion makes important the particle production at the sources of very high energy cosmic rays where the VHE interactions with positive matter target may take place.
\end{abstract}

\section{Introduction}

The spectra of baryons at the LHC can explain the features of cosmic ray particle spectra at very high energies. The transverse momentum distributions are the primary data that can be obtained in the study of hadron spectra at modern colliders. Interpretation of these distributions in up-to-date phenomenological models can shed a light on the physics of hadroproduction processes at high energies. The phenomenological approach is applied here to the description of $p_{T}$ spectrum of various sorts of baryons in the framework of Quark-Gluon String Model [1]. The model has described the data of previous colliders up to energies $\sqrt{(s)}=53 \mathrm{GeV}$ at the area of low $p_{T}$ that gives main contribution to the average value of transverse momenta [2]. Recently $\Lambda^{0}$ production has been studied [3] in an updated version of this model.

The complete study of baryon spectra at LHC energies $(\sqrt{(} s)=0.9-7 \mathrm{TeV})$ [4] did not show important changes that may be responsible for the "knee" in CR proton spectra, see Fig. 1.

It was observed that the average $p_{T}$ of baryons is slowly growing with energy. The average transverse momenta dependence on the mass of baryon (meson) at the LHC energy is also considered up to the masses of charmed and beauty hadrons.

It is suggested in QGSM as well that the explanation of growing charge ratios of secondary antiparticle-toparticle spectra in cosmic rays can be done with the leading production asymmetry of baryon spectra toward

a e-mail: piskoun@sci.lebedev.ru the antibaryon spectra in the kinematical region of fragmentation of targets, which are mostly of positive matter. The procedure of spectra transfer from center-ofmass system at LHC p-p collisions into the laboratory system at cosmic ray interactions is given in the Appendix.

\section{QGSM model for the production of baryons}

Let us first describe the QGSM approach, which has been applied for recent studies of $\Lambda^{0}$. According to this approach baryon production can be parameterized in the following way:

$$
\frac{d \sigma}{p_{T} d p_{T}}=A_{0} \exp \left[-B_{0} \cdot\left(m_{T}-m_{0}\right)\right],
$$

where, $\left.m_{T}=\sqrt{(} p_{T}^{2}+m_{0}^{2}\right), m_{0}$ is the mass of the produced hadron and $B_{0}$ is the slope parameter for the considered energy. In the early paper [3], it was also shown that the value of the slope parameter $B_{0}$ becomes dependent on the collision energy. This approximation works well up to $p_{T}=6 \mathrm{GeV}$, that gives $85 \%$ of integral cross section.

\section{Average transverse momenta of baryons at LHC}

Let us now discuss the mean transverse momenta of produced baryons [4] and look at its dependence on the collision energy, $\sqrt{s}$. It is reasonable also to compare

This is an Open Access article distributed under the terms of the Creative Commons Attribution License 4.0, which permits unrestricted use, distribution, and reproduction in any medium, provided the original work is properly cited. 


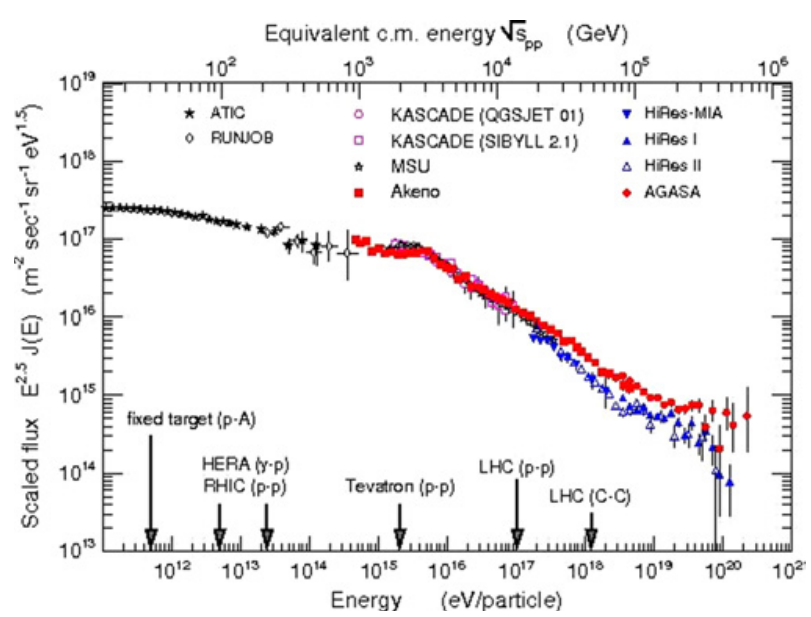

Figure 1. The cosmic proton spectrum with the "knee" between Tevatron and LHC energies.

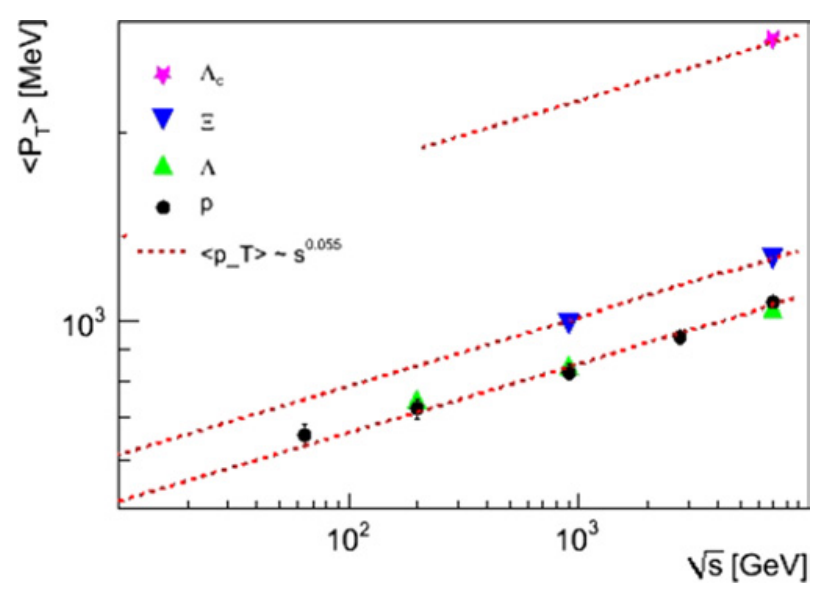

Figure 2. Mean transverse momenta of charged baryons [5-8] as a function of $\sqrt{s}$. Lines show the power-law dependence $s^{0.055}$.

the values calculated for the proton spectra with other available data on baryon production: $\Lambda, \Xi[5]$ and $\Lambda_{c}$ [8] spectra. Figure 2 shows such dependence for the available experimental data. The steep rise of the mean transverse momenta $<p_{T}>$ with energy is seen in Fig. 2. Remarkably, this rise can be parameterized by the same power-like $s^{0.055}$ behavior in the case for all the species of produced baryons.

Another interesting implication reveals in the comparison of the average transverse momenta of various produced baryons at certain collision energies as a function of their masses, shown in Fig. 3. There a linear dependence between the mean transverse momenta $\left\langle p_{T}\right\rangle$ and the baryon mass $M$ is observed. At $\sqrt{s}=7 \mathrm{TeV}$ the average transverse momentum reaches the value of baryon mass, i.e. $\left\langle p_{T}>\sim M\right.$. Further measurements at LHC Run-II should clarify whether or not the average transverse momentum expansion in the baryon production has a limit $<p_{T}>=M$.

It also would be interesting to compare $<p_{T}>$ as a function of produced baryon mass, $M$, with the mass dependence of the average transverse momenta that have been calculated from the description of charged meson production.

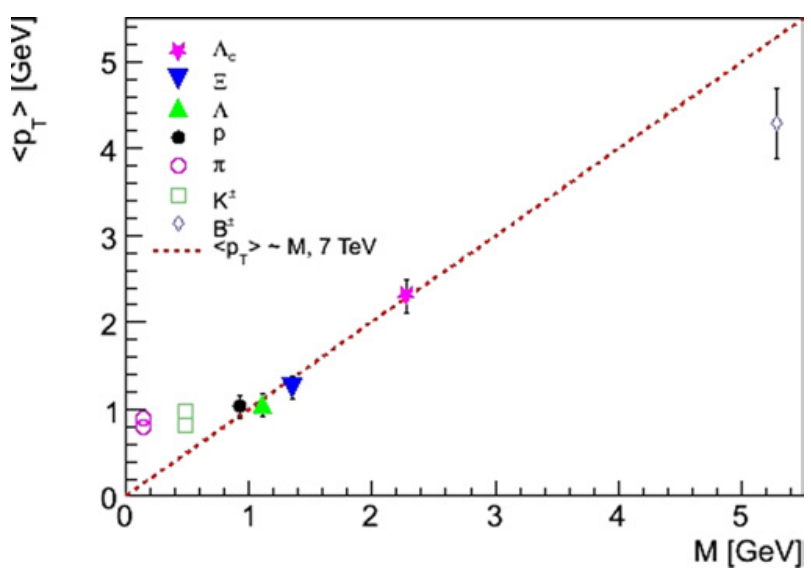

Figure 3. Average transverse momenta of baryons $[5,7,8]$ as a function of their mass are presented at the same energy. Red dashed line shows linear dependence.

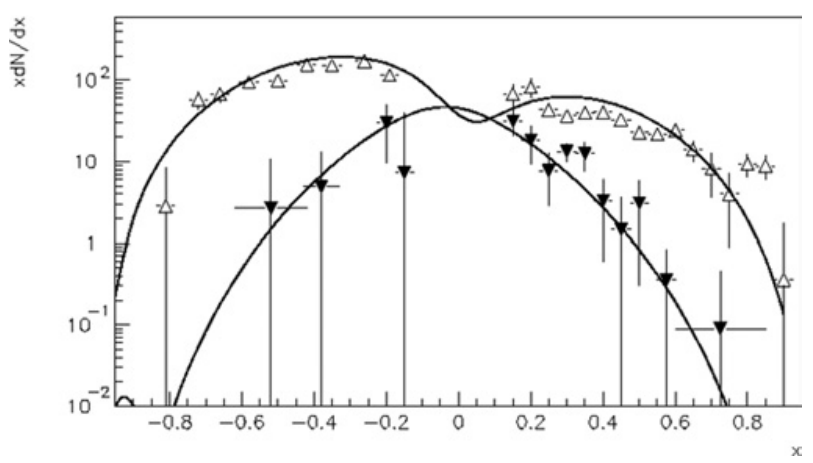

Figure 4. The form of energy spectra of $\Lambda^{0}$ produced in hyperonproton collisions as compiled from two fix target experiments [9].

\section{Antiparticle-to-particle ratios as a result of leading baryon asymmetry}

The important feature on baryon production in protonproton interactions is valuable asymmetry between antibaryon and baryon energy distributions in the kinematical regions of beam fragmentation. It is seen in Fig. 4, where the spectra of $\Lambda_{c}$ are described in QGSM for the entire kinematical $x_{F}$ range [9].

Larger asymmetry appears in the spectra of protons and antiprotons as seen in Fig. 5. What is also important, there is a dip between the growing central part of distribution (the "table") and the stable proton fragmentation region. Such a whimsical form of c.m.s. spectra would give "knees" and "shoulders" at the end of spectra in laboratory system of cosmic ray interactions.

In the laboratory system of cosmic ray interactions, the spectra are to be converted into the energy distributions like those shown in Fig. 6, where the valuable asymmetry takes place at the energies near proton mass. The procedure of transformation of spectra at the pass from center-ofmass system into laboratory system of coordinates is presented in the Appendix.

The growing ratio between antiparticle and particle spectra appears in the left side of spectra due to influence of positive proton target, as was already learned from an early publication [11]. 


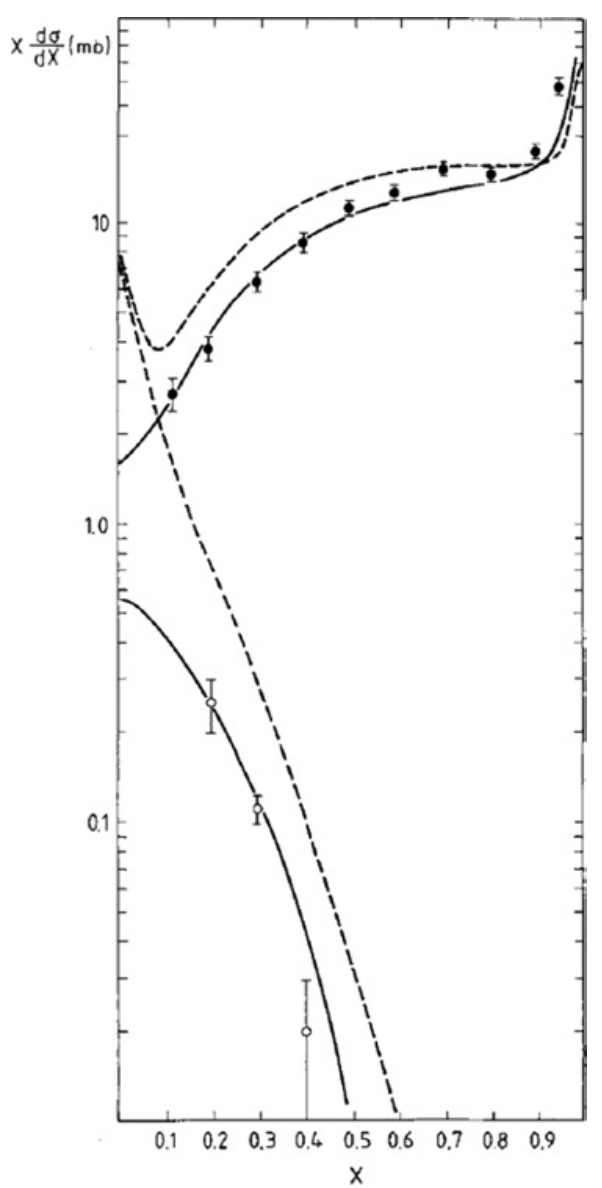

Figure 5. Spectra of protons (black circles) and antiprotons (empty circles) in the low energy experiment and the spectra expected at high energy [1].

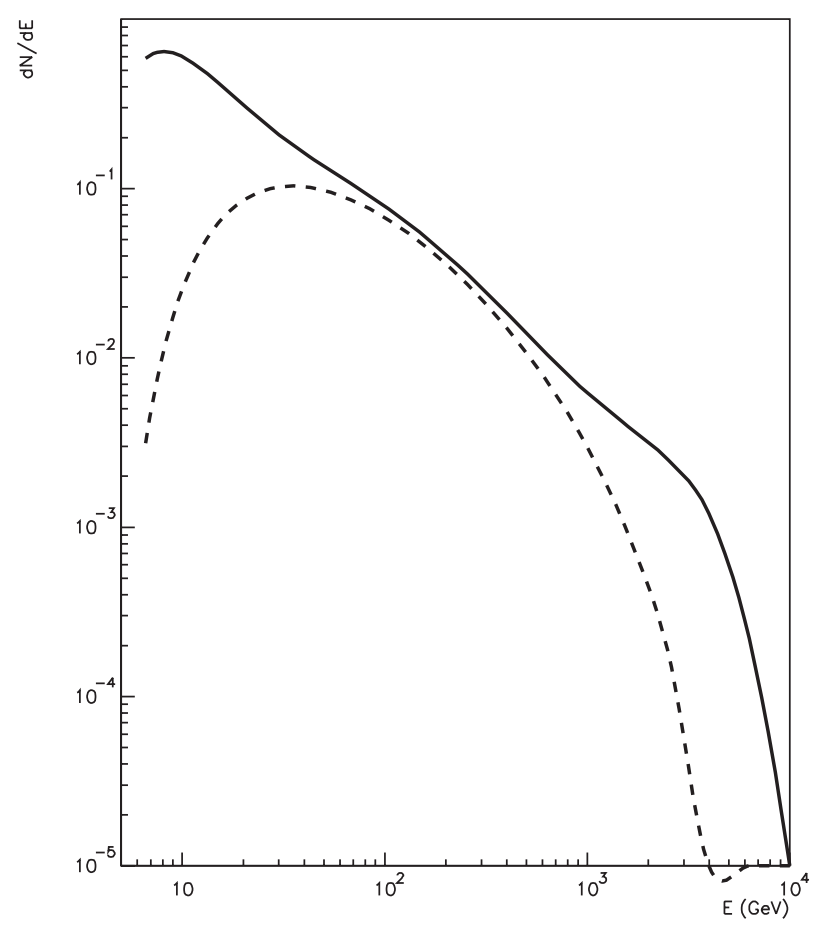

Figure 6. The form of energy spectra of $\Lambda_{c}$ baryons produced in the proton-proton collisions and recalculated into the laboratory system in [10].

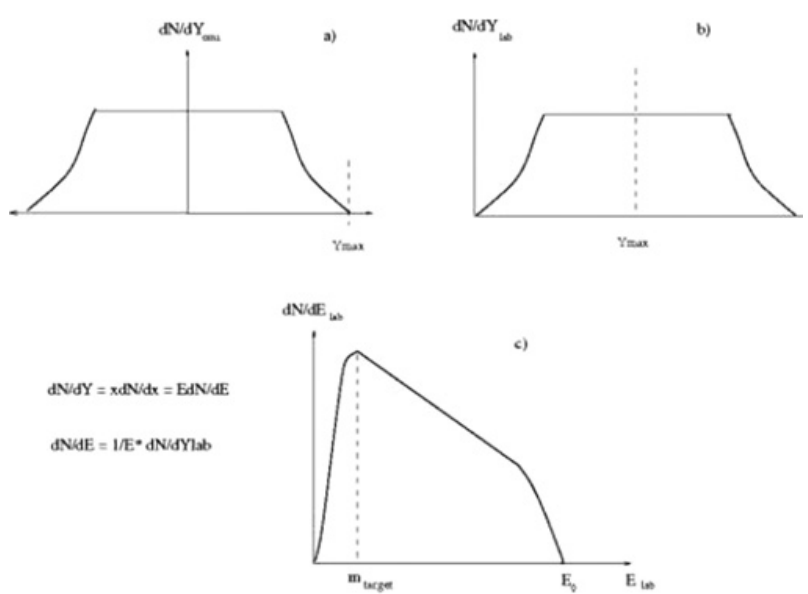

Figure 7. The graphical procedure of recalculation of baryon $\mathrm{dN} / \mathrm{dY}$ spectra measured in c.m. system at collider experiments into the spectra $\mathrm{dN} / \mathrm{dE}$, as they are seen in the laboratory system at cosmic ray measurements. This procedure was first used in [12].

This conclusion makes the particle production at the sources of very high energy cosmic rays important.

\section{Conclusions}

Transverse momentum spectra of protons and antiprotons from RHIC $(\sqrt{(} s)=62$ and $200 \mathrm{GeV})$ and LHC experiments $(\sqrt{(s)}=0.9$ and $7 \mathrm{TeV})$ have been described in the QGSM approach. This model seems to work for the up-todate collider energies, because spectra at low $p_{T}$ are still giving the main part of integral cross section. It seems that the enhancement of the power-law contribution into the spectra at high $p_{T}$ causes the change of low $p_{T}$ exponential slopes, so that the mean transverse momenta are growing with energy. These average transverse momenta are also growing with mass if we analyze the spectra of different baryons independent of their masses. Nevertheless, all these changes in hadroproduction spectra cannot be dramatic enough to cause the "knee" in the primary proton spectra in cosmic rays at the energy in the laboratory system correspondent to the LHC energy. Another aspect of application of QGSM approach is charge asymmetry of CR spectra. The growing antiparticle/particle ratios cannot be the result of acceleration of matter that is mostly positive around us. Growing charge ratios of secondary particle spectra in CR may be generated by the baryon production in the $\mathrm{CR}$ interactions with positive matter targets.

\section{Appendix}

It was found in an earlier paper [12] that the typical rapidity distributions of hadrons at collider experiments are easily convertible into $\mathrm{dN} / \mathrm{dE}$ energy spectra in the laboratory system, where one beam particle becomes the target. This procedure is graphically illustrated in Fig. 7.

As we know the rapidity distribution, $\mathrm{dN} / \mathrm{dY}$, at high energy proton collisions looks like the "table". Rapidity spectrum in laboratory system are reached by the shift of the "table" on the value of $Y_{\max }$, so that all rapidity range is 
positive as in fixed target collisions. Then our distribution should be expressed in energy variable $\mathrm{x}=\mathrm{E} / E_{\text {collision }}$ : $\mathrm{dN} / \mathrm{dY}=\mathrm{xdN} / \mathrm{dx}$. It is clear that the energy spectrum in the laboratory system will be power like $\mathrm{dN} / \mathrm{dE} \sim 1 / \mathrm{E}$.

The author is sincerely thankful to Prof. K. Boreskov for numerous discussions and useful advice.

\section{References}

[1] A.B. Kaidalov and O.I. Piskunova, Z. Phys. C 30 (1986) 145

[2] A.I. Veselov, O.I. Piskunova, K.A. Ter-Martirosian, Phys. Lett. B 158 (1985) 175

[3] O.I. Piskounova, e-print:arXiv 1405.4398, submitted to Conf. series of IOP science, 2014

[4] A. Bylinkin and O.I. Piskounova To be published in the ICHEP14 Proceedings
[5] V. Khachatryan et al. [CMS Collaboration], JHEP 1105 (2011) 064 [arXiv:1102.4282 [hep-ex]]

[6] A. Adare et al. [PHENIX Collaboration], Phys. Rev. C 83 (2011) 064903 [arXiv:1102. 0753 [nucl-ex]]

[7] V. Khachatryan et al. [CMS Collaboration], Phys. Rev. Lett. 105 (2010) 022002 [arXiv:1005.3299 [hep-ex]]

[8] R. Aaij et al. [LHCb Collaboration], Nucl. Phys. B 871 (2013) 1 [arXiv:1302. 2864 [hep-ex]]

[9] Olga I. Piskounova (Lebedev Inst.), Phys. Atom. Nucl. 66 (2003) 307-312, e-Print: hep-ph/0202005 | PDF

[10] O. I. Piskounova (Lebedev Inst.), N. V. Nikitin (SINP, Moscow), Phys. Atom. Nucl. 68 (2005) 2124, Yad. Fiz. 68 (2005) 2186-2190, e-Print: hep-ph/0503006 | PDF

[11] O. I. Piskunova, Sov. J. Nucl. Phys. 47 (1988) 480

[12] O.I. Piskunova, Sov. J. Nucl. Phys. 51 (1990) 846 